\title{
Existence of Multiple Positive Solutions of a Type of Impulsive Functional Differential Equations
}

\author{
Luxia Li, Yuanhua Qiao (Corresponding author), Haili Wang \\ College of Applied Sciences, Beijing University of Technology \\ Ping Le Yuan 100, Beijing 100124, China
}

Tel: 86-10-6739-2182 E-mail: qiaoyuanhua@bjut.edu.cn

Received: October 20, 2011 Accepted: November 4, 2011 Published: February 1, 2012

doi:10.5539/jmr.v4n1p78 URL: http://dx.doi.org/10.5539/jmr.v4n1p78

The research is financed by Natural Science Foundation of China. No.61070149.

\begin{abstract}
In this paper, we consider the non-periodic boundary value problem for a type of first order impulsive functional differential equation in Banach spaces. The existence of pulse in differential equations makes them an important area of investigation. We make use of fixed point index theory on the cone to prove existence of positive solutions. The conditions for existence of two and three positive solutions are given.
\end{abstract}

Keywords: Pulse equation, Periodic boundary value problems, Positive solution

\section{Introduction and Preliminaries}

In recent years, the theories of impulsive functional differential equations have been rapidly developed, and because such equations may exhibit several real world phenomena in physics, biology, engineering, and so forth (Bainov \& Simeonov, 1993; Lakshmikantham, Bainov \& Simeonov, 1989; Bainov \& Hristova, 1993), they have received much attention (Ding, Mi \& Han, 2005; Zhang \& Liu, 2010), The periodic boundary value problem is an important research branch of the impulsive functional differential equations. Some conclusions have been made (Zhang, Li, Jiang \& Wang, 2006; Zhimin \& Weigao, 2002) about the existence of solutions and the multiplicity of positive solutions of the impulsive functional differential equations with periodic boundary value problems. Whereas the non periodic boundary value occurs more frequently in differential equations with pulse, researches are needed for the problem of existence of positive solutions and multiplicity of such equations. In this paper£we restrict our attention to the study of the following first order impulsive functional differential equations with non-periodic boundary value

$$
\left\{\begin{array}{l}
u^{\prime}(t)+M^{2} u(t)=f\left(t, u_{t}\right), \quad t \in J=[0, T], \quad t \neq t_{k}, k=1,2, \cdots, m \\
\Delta u\left(t_{k}\right)=I_{k}\left(u_{t_{k}}\right), \quad k=1, \cdots, m \\
u(0)=p u(T), \\
u(t)=u(0), \quad t \in[-\tau, 0]
\end{array}\right.
$$

Where, $f: J \times C_{\tau}$ is continuous. $C_{\tau}:=\{\varphi:[-\tau, 0] \rightarrow R ; \varphi(t)$ is continuous everywhere except a finite number of points $\bar{t}, \varphi\left(\bar{t}^{+}\right), \varphi\left(\bar{t}^{-}\right)$exist, and $\left.\varphi(\bar{t})=\varphi\left(\bar{t}^{-}\right)\right\} . \tau>0$ is a constant. $u_{t} \in C_{\tau}, u_{t}(\theta)=u(t+\theta), \theta \in[-\tau, 0], 0<t_{1}<t_{2}<\cdots<t_{m}<$ $T, J^{\prime}=J \backslash\left\{t_{1}, t_{2}, \cdots, t_{m}\right\}, I_{k} \in C\left(C_{\tau}, R\right), \Delta u\left(t_{k}\right)=u\left(t_{k}^{+}\right)-u\left(t_{k}^{-}\right)$indicates the jump at $t=t_{k}, u\left(t_{k}^{+}\right)$and $u\left(t_{k}^{-}\right)$indicate the left limit and the right limit of $u(t)$ at $t=t_{k}, J^{*}=[-\tau, T], p \in R$. For $\varphi \in C_{\tau}$, its norm is defined as $\|\varphi\|_{[-\tau, 0]}=\max \theta \in[-\tau, 0]|\varphi(\theta)|$.

The approaches used for the investigation of existence of positive solutions for differential equations with impulse are monotone iterative technique and upper and lower solution method (Zhimin \& She, 2002; Juan \& Rosana, 2006; Luo \& Jing, 2008; He \& He, 2004). Upper and lower solution method is often applied to discuss the minimal and maximal solutions of such equations, and monotone iterative technique is usually used to prove the existence of solution. Recently fixed point index theorem on cones in Banach space is introduced to investigate the multiplicity of solutions (Zhao, 2010). In (Zhao, 2010), Zhao studied the problem (1), the results are established using the fixed point index theorem on the cone, and they proved the existence of two solutions.

Motivated by the results mentioned above, in this paper, we give the conditions of the existence of two positive solutions and three positive solutions of equations (1) using fixed point index theory on the cone.

\section{Preliminaries}

Throughout the rest of this paper, we always assume that the points of impulse $t_{k}$ are right-dense for each $k=$ 
$1,2, \cdots, m . t \in J^{\prime}=J \backslash\left\{t_{1}, t_{2}, \cdots, t_{m}\right\}$.

We define $P C\left(J^{*}\right)=\left\{u: J^{*} \rightarrow R\right.$ is continuous everywhere except a finite number of points in $[-\tau, 0]$, for $t \in J^{\prime}=J \backslash$ $\left\{t_{1}, t_{2}, \cdots, t_{m}\right\}, u\left(t_{k}^{+}\right)$and $u\left(t_{k}^{-}\right)$exist, and $\left.u\left(t_{k}^{-}\right)=u\left(t_{k}\right), k=1,2, \cdots, m\right\}$. Let $E_{0}=\left\{u \in P C\left(J^{*}\right): u(t)=u(0), t \in[-\tau, 0]\right\}$ with the norm $\|u\|_{[-\tau, T]}=\sup _{t \in J^{*}}|u(t)|$, then $E_{0}$ is a Banach space. Let $C_{\tau}^{+}=\left\{\varphi \in C_{\tau}: \varphi(\theta) \geq 0, \theta \in[-\tau, 0]\right\}$, $C^{*}=\left\{\varphi \in C_{\tau}: 0 \leq \delta\|\varphi\|_{[-\tau, 0]} \leq \varphi(\theta), \theta \in[-\tau, 0]\right\}$.

Theorem 1.1 Suppose that $E$ is a Banach space, $K \in E$ is a cone in , and $r>0, \Omega_{r}=\{x \in K:\|x\|<r\}$. If $S: \overline{\Omega_{r}} \rightarrow K$ is a complete continuous operator, and for $\forall x \in \partial \Omega_{r}, S x \neq x$

(1) if $\|S x\| \leq\|x\|, \forall x \in \partial \Omega_{r}$, then $i\left(S, \Omega_{r}, K\right)=1$;

(2) if $\|S x\| \geq\|x\|, \forall x \in \partial \Omega_{r}$, then $i\left(S, \Omega_{r}, K\right)=0$.

Lemma $1 u \in E_{0}$ is a solution of question (1), if and only if $u \in E_{0}$ is a solution of the follow integral equation:

$$
u(t)= \begin{cases}\int_{0}^{T} G(t, s) f\left(s, u_{s}\right) d s+\sum_{k=1}^{m} G\left(t, t_{k}\right) I_{k}\left(u_{t_{k}}\right), & t \in[0, T] \\ u(0), & t \in[-\tau, 0]\end{cases}
$$

Where

$$
G(t, s)=\frac{1}{e^{M^{2} T}-p}\left\{\begin{array}{cc}
p e^{M^{2}(s-t)} & , \quad 0 \leq t \leq s \leq T \\
p e^{M^{2}(T-t+s)} & , \quad 0 \leq s \leq t \leq T
\end{array}\right.
$$

For convenient, we always suppose that:

$\left(H_{1}\right) 0<p<e^{M^{2} T}$;

$\left(H_{2}\right) f$ is an impulsive $L^{1}-$ Caratheodory function, and for almost all of $t \in J^{\prime}=J \backslash\left(t_{1}, t_{2}, \cdots, t_{m}\right)$ and $v \in C_{\tau}^{+}, f(t, v) \geq 0$;

$\left(H_{3}\right) I_{k}(k=1,2, \cdots, m)$ is continuous, and for any $v \in C_{\tau}^{+}, I_{k}(v) \geq 0$.

We define an operator $S: E_{0} \rightarrow E_{0}$

$$
S(u)(t)= \begin{cases}F(u)(t), & t \in[0, T] \\ F(u)(0), & t \in[-\tau, 0]\end{cases}
$$

Where

$$
F(u)(t)=\int_{0}^{T} G(t, s) f\left(s, u_{s}\right) d s+\sum_{k=1}^{m} G\left(t, t_{k}\right) I_{k}\left(u_{t_{k}}\right), \quad t \in J
$$

Then $u \in E_{0}$ is a solution of problem (2) if and only if $u \in E_{0}$ is a fixed point of operator $S$. We define a cone $K$ in Banach space $E_{0}$ as follows

$$
K=\left\{u \in E_{0}: u(t) \geq \delta\|u\|_{[-\tau, T]}, t \in J^{*}\right\}
$$

Where $\delta=\frac{\min \{1, p\}}{e^{M^{2} T} \max \{1, p\}}$.

Lemma 2 If conditions $\left(H_{1}\right),\left(H_{2}\right),\left(H_{3}\right)$ hold, then $S(K) \subset K$, and $S: K \rightarrow K$ is completely continuous.

Lemma 3 If conditions $\left(H_{1}\right),\left(H_{2}\right),\left(H_{3}\right)$ hold, and $f(t, 0) \neq 0, \quad I_{k}(0) \neq 0$, then $S(0) \neq 0$.

\section{Conclusions}

Theorem 3.1 Suppose conditions $\left(H_{1}\right),\left(H_{2}\right),\left(H_{3}\right)$ hold, and as well as the following conditions $\left(H_{4}\right)$ and $\left(H_{5}\right)$.

$\left(H_{4}\right) \lim _{v \in C^{*},\|v\|_{[-\tau, 0]} \rightarrow 0} \frac{f(t, v)}{\|v\|_{[-\tau, 0]}}=+\infty$ and $\lim _{v \in C^{*},\|v\|_{[-\tau, 0]} \rightarrow \infty} \frac{f(t, v)}{\|v\|_{[-\tau, 0]}}=+\infty$ hold conformably for all $t \in[0, T]$.

$\left(H_{5}\right)$ There exit constants $d>0, \eta, \eta_{k} \geq 0$ satisfying

$$
\max \left\{f(t, v): t \in J, \delta d \leq\|v\|_{[-\tau, 0]} \leq d, v \in C^{*}\right\}<\eta d .
$$

and

$$
\max \left\{I_{k}(v): \delta d \leq\|v\|_{[-\tau, 0]} \leq d, v \in C^{*}\right\}<\eta_{k} d, k=1,2, \cdots, m
$$

Where $\eta$ and $\eta_{k}$ satisfy

$$
\eta+\sum_{k=1}^{m} \eta_{k}>0, \max \left\{\eta \int_{0}^{T} G(t, s) d s+\sum_{k=1}^{m} G\left(t, t_{k}\right) \eta_{k}\right\}<\delta
$$

Then problem (1) has at least two positive solutions $u_{1}, u_{2}$, which satisfy $0<\left\|u_{1}\right\|_{[-\tau, T]}<d<\left\|u_{2}\right\|_{[-\tau, T]}$. 
Proof: Following condition $\left(H_{4}\right)$, and take $N>\left(\delta \min _{t \in[0, T]} \int_{0}^{T} G(t, s) d s\right)^{-1}$, then there exist constants $r>0, R>0(r$ is sufficiently small, and $R$ is sufficiently large, $r<d<R$ ), for $\forall t \in[0, T]$, we have:

$$
f(t, v)>N\|v\|_{[-\tau, 0]}, \quad v \in C^{*}, \quad\|v\|_{[-\tau, 0]} \leq r
$$

and

$$
f(t, v)>N\|v\|_{[-\tau, 0]}, \quad v \in C^{*},\|v\|_{[-\tau, 0]} \geq \delta R
$$

Let $\Omega_{r}=\left\{u \in E_{0}:\|u\|_{[-\tau, T]}<r\right\}, \Omega_{R}=\left\{u \in E_{0}:\|u\|_{[-\tau, T]}<R\right\}$.

For $\forall u \in K \cap \partial \Omega_{r}$ and $\forall t \in J, \delta\|u\|_{[-\tau, T]} \leq\left\|u_{t}\right\|_{[-\tau, 0]} \leq\|u\|_{[-\tau, T]}=r$ holds, and

$$
0<\delta\left\|u_{t}\right\|_{[-\tau, 0]} \leq \delta\|u\|_{[-\tau, T]} \leq u(t+\theta)=u_{t}(\theta)
$$

Therefore $u_{t} \in C^{*}$. According to condition $\left(H_{4}\right)$ and inequality (6), we have $f\left(t, u_{t}\right)>N\left\|u_{t}\right\|_{[-\tau, 0]}$. Consequently, if $t \in J$, then

$$
\begin{aligned}
S(u)(t) & =\int_{0}^{T} G(t, s) f\left(s, u_{s}\right) d s+\sum_{k=1}^{m} G\left(t, t_{k}\right) I_{k}\left(u_{t_{k}}\right) \\
& \geq \int_{0}^{T} G(t, s) N\left\|u_{s}\right\|_{[-\tau, 0]} d s \\
& \geq\left(N \delta \min _{t \in[0, T]} \int_{0}^{T} G(t, s) d s\right)\|u\|_{[-\tau, T]} \\
& >\|u\|_{[-\tau, T]}
\end{aligned}
$$

Therefore for $\forall u \in K \cap \partial \Omega_{r}$, we have $\|S u\| \geq\|u\|_{[-\tau, T]}=r$.

For $\forall u \in K \cap \partial \Omega_{R}$ and $\forall t \in J$ we have $\left\|u_{t}\right\|_{[-\tau, 0]} \geq \delta\|u\|_{[-\tau, T]}=\delta R$, and $0<\delta\left\|u_{t}\right\|_{[-\tau, 0]} \leq u_{t}(\theta)$.

Therefore $\forall u \in C^{*}$. According to condition $H_{4}$ and inequality (7), we have $f\left(t, u_{t}\right)>N\left\|u_{t}\right\|_{[-\tau, 0]}$.

Therefore when $t \in J$,

$$
\begin{aligned}
S(u)(t) & =\int_{0}^{T} G(t, s) f\left(s, u_{s}\right) d s+\sum_{k=1}^{m} G\left(t, t_{k}\right) I_{k}\left(u_{t_{k}}\right) \\
& \geq \int_{0}^{T} G(t, s) N\left\|u_{s}\right\|_{[-\tau, 0]} d s \\
& \geq\left(N \delta \min _{t \in[0, T]} \int_{0}^{T} G(t, s) d s\right)\|u\|_{[-\tau, T]} \\
& >\|u\|_{[-\tau, T]}
\end{aligned}
$$

and $\|S u\| \geq\|u\|_{[-\tau, T]}=R$ holds.

Let $\Omega_{d}=\left\{u \in E_{0}:\|u\|_{-\tau, T}<d\right\}$, according to condition $V$, for $\forall u \in K \bigcap \partial \Omega_{d}, \forall t \in[0, T]$ we have $u_{t} \in C^{*}$ and $\delta d=\delta\|u\|_{[-\tau, T]} \leq\|u\|_{[-\tau, 0]} \leq\|u\|_{[-\tau, T]}=d$. Consequently,

$$
\max \left\{f\left(t, u_{t}\right)\right\}<\eta d, \max \left\{I_{k}\left(u_{t}\right)\right\}<\eta_{k} d, \quad \forall t \in J
$$

Therefore

$$
\begin{aligned}
S(u)(t) & =\int_{0}^{T} G(t, s) f\left(s, u_{s}\right) d s+\sum_{k=1}^{m} G\left(t, t_{k}\right) I_{k}\left(u_{t_{k}}\right) \\
& \leq \int_{0}^{T}(G(t, s) \eta d) d s+\sum_{k=1}^{m} G\left(t, t_{k}\right) \eta_{k} d \\
& \leq \max _{t \in[0, T]}\left\{\int_{0}^{T} \eta G(t, s) d s+\sum_{k=1}^{m} G\left(t, t_{k}\right) \eta_{k}\right\} d \\
& <\delta d=\delta\|u\|_{[-\tau, T]} \leq u(t)
\end{aligned}
$$


Therefore for $\forall u \in K \cap \partial \Omega_{d}$ and $\forall t \in[0, T]$ we have

$$
\|S u\| \leq\|u\|_{[-\tau, T]}=d \quad \text { and } \quad S(u)(t) \neq u(t) .
$$

According to theorem 1.1, we have that $S$ exits at least two fixed points $u_{1}, u_{2}$ and $u_{1} \in K \bigcap\left(\Omega_{d} \backslash \Omega_{r}\right), u_{2} \in K \cap\left(\overline{\Omega_{R}} \backslash \overline{\Omega_{d}}\right)$. It means that we have at least two positive solutions $u_{1}, u_{2}$ for problem (1) and they satisfy inequality $0<\left\|u_{1}\right\|_{[-\tau, T]}<$ $d<\left\|u_{2}\right\|_{[-\tau, T]}$.

Theorem 3.2 Suppose conditions $\left(H_{1}\right),\left(H_{2}\right),\left(H_{3}\right)$ hold, together with the following conditions $\left(H_{6}\right)$ and $\left(H_{7}\right)$. $\left(H_{6}\right)$

and

$$
\lim _{v \in C^{*},\|v\|_{[-\tau, 0]} \rightarrow 0} \frac{f(t, v)}{\|v\|_{[-\tau, 0]}}=0, \quad \lim _{v \in C^{*},\|v\|_{[-\tau, 0]} \rightarrow 0} \frac{I_{k}(v)}{\|v\|_{[-\tau, 0]}}=0,
$$

$$
\lim _{v \in C^{*},\|v\|_{[-\tau, 0]} \rightarrow \infty} \frac{f(t, v)}{\|v\|_{[-\tau, 0]}}=0, \quad \lim _{v \in C^{*},\|v\|_{[-\tau, 0]} \rightarrow \infty} \frac{I_{k}(v)}{\|v\|_{[-\tau, 0]}}=0
$$

hold uniformly for all $t \in[0, T]$.

$\left(H_{7}\right)$ There exit constants $d>0, \eta, \eta_{k} \geq 0$ such that

$$
\min \left\{f(t, v): t \in J, \delta d \leq\|v\|_{[-\tau, 0]} \leq d, v \in C^{*}\right\}>\eta d .
$$

and

$$
\min \left\{I_{k}(v): \delta d \leq\|v\|_{[-\tau, 0]} \leq d, v \in C^{*}\right\}>\eta_{k} d, k=1,2, \cdots, m
$$

Where $\eta, \eta_{k}$ satisfy

$$
\eta+\sum_{k=1}^{m} \eta_{k}>0, \min \left\{\eta \int_{0}^{T} G(t, s) d s+\sum_{k=1}^{m} G\left(t, t_{k}\right) \eta_{k}\right\}>1
$$

Then problem (1) has at least two positive solutions $u_{1}$, $u_{2}$, which satisfy $0<\left\|u_{1}\right\|_{[-\tau, T]}<d<\left\|u_{2}\right\|_{[-\tau, T]}$.

Proof: Take $\xi, \xi_{k}>0$ sufficiently small such that

$$
\max \left\{\xi \int_{0}^{T} G(t, s) d s+\sum_{k=1}^{m} G\left(t, t_{k}\right) \xi_{k}\right\}<\delta .
$$

From condition $\left(H_{6}\right)$, there exists constants $r>0, R>0(r$ is sufficiently small, and $R$ is sufficiently large, $r<d<R)$, for $\forall t \in[0, T]$, we have:

$$
f(t, v)<\xi\|v\|_{[-\tau, 0]}, \quad I_{k}(v)<\xi k\|v\|_{[-\tau, 0]}, \quad v \in C^{*},\|v\|_{[-\tau, 0]} \leq r
$$

and

$$
f(t, v)<\xi\|v\|_{[-\tau, 0]}, \quad I_{k}(v)<\xi_{k}\|v\|_{[-\tau, 0]}, \quad v \in C^{*},\|v\|_{[-\tau, 0]} \geq \delta R
$$

Let $\Omega_{r}=\left\{u \in E_{0}:\|u\|_{[-\tau, T]}<r\right\}, \Omega_{R}=\left\{u \in E_{0}:\|u\|_{[-\tau, T]}<R\right\}$.

For $\forall u \in K \cap \partial \Omega_{r}$ and $\forall t \in J, \delta\|u\|_{[-\tau, T]} \leq\left\|u_{t}\right\|_{[-\tau, 0]} \leq\|u\|_{[-\tau, T]}=r$ and

$$
0<\left\|u_{t}\right\|_{[-\tau, 0]} \leq \delta\|u\|_{[-\tau, T]} \leq u(t+\theta)=u_{t}(\theta)
$$

Therefore we assert $u_{t} \in C^{*}$. According to condition $\left(H_{6}\right)$ and inequality (8), we have

$$
f\left(t, u_{t}\right)<\xi\left\|u_{t}\right\|_{[-\tau, 0]}, \quad I_{k}\left(u_{t}\right)<\xi_{k}\left\|u_{t}\right\|_{[-\tau, 0]}
$$

Consequently, for $t \in J$

$$
\begin{aligned}
S(u)(t) & =\int_{0}^{T} G(t, s) f\left(s, u_{s}\right) d s+\sum_{k=1}^{m} G\left(t, t_{k}\right) I_{k}\left(u_{t_{k}}\right) \\
& \leq \int_{0}^{T} G(t, s) \xi\left\|u_{s}\right\|_{[-\tau, 0]} d s+\sum_{k=1}^{m} G\left(t, t_{k}\right) \xi_{k}\left\|u_{t_{k}}\right\|_{[-\tau, 0]} \\
& \leq \max _{t \in[0, T]}\left\{\xi \int_{0}^{T} G(t, s) d s+\sum_{k=1}^{m} G\left(t, t_{k}\right) \xi_{k}\right\}\|u\|_{[-\tau, T]} \\
& <\delta\|u\|_{[-\tau, T]} \leq u(t)
\end{aligned}
$$


Therefore for $u \in K \cap \partial \Omega_{r}$

$$
\|S u\| \leq\|u\|_{[-\tau, T]}=r \quad \text { and } \quad S(u)(t) \neq u(t) \quad \text { hold. }
$$

For $\forall u \in K \bigcap \partial \Omega_{R}$ and $\forall t \in J$, we have

$$
\left\|u_{t}\right\|_{[-\tau, 0]} \geq \delta\|u\|_{[-\tau, T]}=\delta R \quad \text { and } \quad 0<\delta\left\|u_{t}\right\|_{[-\tau, 0]} \leq u_{t}(\theta)
$$

hereby $u_{t} \in C^{*}$, according to condition $\left(H_{6}\right)$ and inequality (9), we have

$$
f\left(t, u_{t}\right)<\xi\left\|u_{t}\right\|_{[-\tau, 0]}, \quad I_{k}\left(u_{t}\right)<\xi_{k}\left\|u_{t}\right\|_{[-\tau, 0]}
$$

Consequently, for $t \in J$, we have

$$
\begin{aligned}
S(u)(t) & =\int_{0}^{T} G(t, s) f\left(s, u_{s}\right) d s+\sum_{k=1}^{m} G\left(t, t_{k}\right) I_{k}\left(u_{t_{k}}\right) \\
& \leq \int_{0}^{T} G(t, s) \xi\left\|u_{s}\right\|_{[-\tau, 0]} d s+\sum_{k=1}^{m} G\left(t, t_{k}\right) \xi_{k}\left\|u_{t_{k}}\right\|_{[-\tau, 0]} \\
& \leq \max _{t \in[0, T]}\left\{\xi \int_{0}^{T} G(t, s) d s+\sum_{k=1}^{m} G\left(t, t_{k}\right) \xi \xi\|u\|_{[-\tau, T]}\right. \\
& <\delta\|u\|_{[-\tau, T]} \leq u(t)
\end{aligned}
$$

We have $\|S u\| \leq\|u\|_{[-\tau, T]}=R$ and $S(u)(t) \neq u(t)$ hold.

Let $\Omega_{d}=\left\{u \in E_{0}:\|u\|_{[-\tau, T]}<d\right\}$, then for $\forall u \in K \bigcap \partial \Omega_{d}$ and $\forall t \in[0, T]$, according to condition $\left(H_{7}\right)$, we have $u_{t} \in C^{*}$ and $\delta d=\delta\|u\|_{[-\tau, T]} \leq\left\|u_{t}\right\|_{[-\tau, 0]} \leq\|u\|_{[-\tau, T]}=d$, and then

$$
\min \left\{f\left(t, u_{t}\right)\right\}>\eta d, \quad \min \left\{I_{k}\left(u_{t}\right)\right\}>\eta_{k} d \quad \forall t \in J
$$

Then we have

$$
\begin{aligned}
S(u)(t) & =\int_{0}^{T} G(t, s) f\left(s, u_{s}\right) d s+\sum_{k=1}^{m} G\left(t, t_{k}\right) I_{k}\left(u_{t_{k}}\right) \\
& \geq \int_{0}^{T}(G(t, s) \eta d) d s+\sum_{k=1}^{m} G\left(t, t_{k}\right) \eta_{k} d \\
& \geq \min _{t \in[0, T]}\left\{\int_{0}^{T} \eta G(t, s) d s+\sum_{k=1}^{m} G\left(t, t_{k}\right) \eta_{k}\right\} d \\
& >d=\|u\|_{[-\tau, T]}
\end{aligned}
$$

Therefore for $\forall u \in K \cap \partial \Omega_{d}, \forall t \in[0, T]$, we have $\|S u\|>\|u\|_{[-\tau, T]}=d$.

We assert that $S(u)(t) \neq u(t)$.

Therefore from theorem 1.1, we have at least that two fixed points $u_{1}, u_{2}$ for operator $S$. such and $u_{1} \in K \cap\left(\Omega_{d} \backslash \overline{\Omega_{r}}\right)$, and $u_{2} \in K \cap\left(\Omega_{R} \backslash \overline{\Omega_{d}}\right)$.

It means that problem (1) exist at least two positive solutions $u_{1}, u_{2}$, and they satisfy $0<\left\|u_{1}\right\|_{[-\tau, T]}<d<\left\|u_{2}\right\|_{[-\tau, T]}$.

Theorem 3.3 Suppose conditions $\left(H_{1}\right),\left(H_{2}\right),\left(H_{3}\right)$ hold, and $f(t, 0) \neq 0, I_{k}(0) \neq 0$, and with the following conditions $\left(H_{8}\right),\left(H_{9}\right)$ :

$\left(H_{8}\right)$ There exist constants $R>r>0, \xi, \xi_{k}>0$ such that when $v \in C_{\tau}^{+}$, for all of $t \in J$ and $0<\|v\|_{[-\tau, 0]} \leq r$, we have $f(t, v) \leq \xi\|v\|_{[-\tau, 0]}, \quad I_{k}(v) \leq \xi_{k}\|v\|_{[-\tau, 0]}$ and $\xi+\xi_{k}>0, \max \left\{\xi \int_{0}^{T} G(t, s) d s+\sum_{k=1}^{m} G\left(t, t_{k}\right) \xi_{k}\right\}<\delta$ hold.

For all of $t \in J$ and $\|v\|_{[-\tau, 0]} \geq \delta R$, we have

$$
f(t, v) \leq \xi\|v\|_{[-\tau, 0]}, I_{k}(v) \leq \xi_{k}\|v\|_{[-\tau, 0]} \text { and } \xi+\sum_{k=1}^{m} \xi_{k}>0, \max \left\{\xi \int_{0}^{T} G(t, s) d s+\sum_{k=1}^{m} G\left(t, t_{k}\right) \xi_{k}\right\}<\delta .
$$


$\left(H_{9}\right)$ There exist constants $r<d<R, \eta, \eta_{k} \geq 0$ such that when $v \in C_{\tau}^{+}$, for all of $t \in J$ and $\delta d \leq\|v\|_{[-\tau, 0]} \leq d$, we have

$$
f(t, v) \geq \eta\|v\|_{[-\tau, 0]}, \quad I_{k}(v) \geq \eta_{k}\|v\|_{[-\tau, 0]} \text { and } \eta+\sum_{k=1}^{m} \eta_{k}>0, \delta \min \left\{\eta \int_{0}^{T} G(t, s) d s+\sum_{k=1}^{m} G\left(t, t_{k}\right) \eta_{k}\right\}>1
$$

Then problem (1) exits at least three positive solutions $u_{1}, u_{2}$, $u_{3}$, and they satisfy $0<\left\|u_{1}\right\|_{[-\tau, T]}<r<\left\|u_{2}\right\|_{[-\tau, T]}<d<$ $\left\|u_{3}\right\|_{[-\tau, T]}<R$.

Proof: For three constants $r<d<R$, we define three open sets:

$$
\Omega_{r}=\left\{u \in E_{0}:\|u\|_{[-\tau, T]}<r\right\}, \Omega_{d}=\left\{u \in E_{0}:\|u\|_{[-\tau, T]}<d\right\}, \Omega_{R}=\left\{u \in E_{0}:\|u\|_{[-\tau, T]}<R\right\} .
$$

Then $\theta \in \Omega_{r} \in \Omega_{d} \in \Omega_{R}$.

For $\forall u \in K \bigcap \partial \Omega_{r}$ and $\forall t \in J$, we have $u_{t} \in C_{\tau}^{+}$and $\delta\|u\|_{[-\tau, T]} \leq\left\|u_{t}\right\|_{[-\tau, 0]} \leq\|u\|_{[-\tau, T]}=r$.

According to condition $\left(H_{8}\right)$, we deduce $f\left(t, u_{t}\right) \leq \xi\left\|u_{t}\right\|_{[-\tau, 0]}$ and $I_{k}\left(u_{t}\right) \leq \xi_{k}\left\|u_{t}\right\|_{[-\tau, 0]}$.

Therefore for $t \in J$

$$
\begin{aligned}
S(u)(t) & =\int_{0}^{T} G(t, s) f\left(s, u_{s}\right) d s+\sum_{k=1}^{m} G\left(t, t_{k}\right) I_{k}\left(u_{t_{k}}\right) \\
& \leq \int_{0}^{T} G(t, s) \xi\left\|u_{s}\right\|_{[-\tau, 0]} d s+\sum_{k=1}^{m} G\left(t, t_{k}\right) \xi_{k}\left\|u_{t_{k}}\right\|_{[-\tau, 0]} \\
& \leq \max _{t \in[0, T]}\left\{\xi \int_{0}^{T} G(t, s) d s+\sum_{k=1}^{m} G\left(t, t_{k}\right) \xi_{k}\right\}\|u\|_{[-\tau, T]} \\
& <\delta\|u\|_{[-\tau, T]} \leq u(t)
\end{aligned}
$$

Accordingly for $\forall u \in K \cap \partial \Omega_{r}$, we have $\|S u\| \leq\|u\|_{[-\tau, T]}=r, \quad S(u)(t) \neq u(t)$.

For $\forall u \in K \cap \partial \Omega_{R}$ and $\forall t \in J$, we have $u_{t} \in C_{\tau}^{+}$and $\left\|u_{t}\right\|_{[-\tau, 0]} \geq \delta\|u\|_{[-\tau, T]}=\delta R$.

According to condition $\left(H_{8}\right)$, we have $f\left(t, u_{t}\right) \leq \xi\left\|u_{t}\right\|_{[-\tau, 0]}, \quad I_{k}\left(u_{t}\right) \leq \xi_{k}\left\|u_{t}\right\|_{[-\tau, 0]}$.

Therefore when $t \in J$

$$
\begin{aligned}
S(u)(t) & =\int_{0}^{T} G(t, s) f\left(s, u_{s}\right) d s+\sum_{k=1}^{m} G\left(t, t_{k}\right) I_{k}\left(u_{t_{k}}\right) \\
& \leq \int_{0}^{T} G(t, s) \xi\left\|u_{s}\right\|_{[-\tau, 0]} d s+\sum_{k=1}^{m} G\left(t, t_{k}\right) \xi_{k}\left\|u_{t_{k}}\right\|_{[-\tau, 0]} \\
& \leq \max _{t \in[0, T]}\left\{\xi \int_{0}^{T} G(t, s) d s+\sum_{k=1}^{m} G\left(t, t_{k}\right) \xi_{k}\right\}\|u\|_{[-\tau, T]} \\
& <\delta\|u\|_{[-\tau, T]} \leq u(t)
\end{aligned}
$$

Then $\|S u\| \leq\|u\|_{[-\tau, T]}=R, \quad S(u)(t) \neq u(t)$.

According to condition $\left(H_{9}\right)$, for $\forall u \in K \cap \partial \Omega_{d}$ and $\forall t \in[0, T]$, we have

$$
u_{t} \in C_{\tau}^{+} \text {and } \delta d=\delta\|u\|_{[-\tau, T]} \leq\left\|u_{t}\right\|_{[-\tau, 0]} \leq\|u\|_{[-\tau, T]}=d
$$

Accordingly $f\left(t, u_{t}\right) \geq \eta\left\|u_{t}\right\|_{[-\tau, 0]}, \quad I_{k}\left(u_{t}\right) \geq \eta_{k}\left\|u_{t}\right\|_{[-\tau, 0]}$ hold for $\forall t \in J$.

Then

$$
\begin{aligned}
S(u)(t) & =\int_{0}^{T} G(t, s) f\left(s, u_{s}\right) d s+\sum_{k=1}^{m} G\left(t, t_{k}\right) I_{k}\left(u_{t_{k}}\right) \\
& \geq \int_{0}^{T} G(t, s) \eta\left\|u_{s}\right\|_{[-\tau, 0]} d s+\sum_{k=1}^{m} G\left(t, t_{k}\right) \eta_{k}\left\|u_{t_{k}}\right\|_{[-\tau, 0]} \\
& \geq \delta \min _{t \in[0, T]}\left\{\eta \int_{0}^{T} G(t, s) d s+\sum_{k=1}^{m} G\left(t, t_{k}\right) \eta_{k}\right\}\|u\|_{[-\tau, T]} \\
& >\|u\|_{[-\tau, T]}
\end{aligned}
$$


Therefore for $\forall u \in K \bigcap \partial \Omega_{d}$ and $\forall t \in[0, T]$, we have $\|S u\|>\|u\|_{[-\tau, T]}=d$, thus $S(u)(t) \neq u(t)$.

Then we deduce from theorem 1.1 that $S$ has at least three fixed points $u_{1}, u_{2}, u_{3}$ such that $u_{1} \in K \cap \Omega_{r}, u_{2} \in K \cap\left(\Omega_{d} \backslash \overline{\Omega_{r}}\right)$, $u_{3} \in K \cap\left(\Omega_{R} \backslash \overline{\Omega_{d}}\right)$.

According to lemma 3, we have $u_{1} \neq 0$.

It means that problem (1) exits at least three positive solutions $u_{1}, u_{2}, u_{3}$ and they satisfy

$$
0<\left\|u_{1}\right\|_{[-\tau, T]}<r<\left\|u_{2}\right\|_{[-\tau, T]}<d<\left\|u_{3}\right\|_{[-\tau, T]}<R .
$$

Theorem 3.4 Suppose conditions $\left(H_{1}\right),\left(H_{2}\right),\left(H_{3}\right)$ hold and $f(t, 0) \neq 0, I_{k}(0) \neq 0$, and the following conditions $\left(H_{10}\right),\left(H_{11}\right)$ also hold:

$\left(H_{10}\right) \lim _{v \in C^{*},\|v\|_{[-\tau, 0]} \rightarrow 0} \frac{f(t, v)}{\|v\|_{[-\tau, 0]}}=0, \quad \lim _{v \in C^{*},\|v\|_{[-\tau, 0]} \rightarrow 0} \frac{I_{k}(v)}{\|v\|_{[-\tau, 0]}}=0, \lim _{v \in C^{*},\|v\|_{[-\tau, 0]} \rightarrow \infty} \frac{f(t, v)}{\|v\|_{[-\tau, 0]}}=0 \quad$ and $\lim _{v \in C^{*},\|v\|_{[-\tau, 0]} \rightarrow \infty} \frac{I_{k}(v)}{\|v\|_{[-\tau, 0]}}=0$, uniformly hold for all of $t \in[0, T]$;

$\left(H_{11}\right)$ There exist constants $d>0, \eta, \eta_{k} \geq 0$, such that

$$
\min \left\{f(t, v): t \in J, \delta d \leq\|v\|_{[-\tau, 0]} \leq d, v \in C^{*}\right\}>\eta d
$$

and

$$
\min \left\{I_{k}(v): \delta d \leq\|v\|_{[-\tau, 0]} \leq d, v \in C^{*}\right\}>\eta_{k} d, \quad k=1,2, \cdots, m
$$

Where $\eta, \eta_{k}$ satisfy

$$
\eta+\sum_{k=1}^{m} \eta_{k}>0, \min \left\{\eta \int_{0}^{T} G(t, s) d s+\sum_{k=1}^{m} G\left(t, t_{k}\right) \eta_{k}\right\}>1
$$

Then problem (1) exits at least three positive solutions $u_{1}, u_{2}, u_{3}$, and they satisfy

$$
0<\left\|u_{1}\right\|_{[-\tau, T]}<r<\left\|u_{2}\right\|_{[-\tau, T]}<d<\left\|u_{3}\right\|_{[-\tau, T]}<R .
$$

Proof: According to conditions $\left(H_{10}\right)$ and $\left(H_{11}\right)$, following the proof of theorem 3.2, we can easily deduce:

for $\forall u \in K \cap \partial \Omega_{r}, \quad\|S u\|>\|u\|_{[-\tau, T]}=r \quad$ and $\quad S(u)(t) \neq u(t)$ hold;

for $\forall u \in K \cap \partial \Omega_{R}, \quad\|S u\|>\|u\|_{[-\tau, T]}=R \quad$ and $\quad S(u)(t) \neq u(t)$ hold;

for $\forall u \in K \cap \partial \Omega_{d}, \quad\|S u\|>\|u\|_{[-\tau, T]}=d, \quad$ so $\quad S(u)(t) \neq u(t)$.

Therefore according to theorem 1.1 we deduce that $S$ has at least three fixed points $u_{1}, u_{2}, u_{3}$ such that $u_{1} \in K \cap \Omega_{r}$, $u_{2} \in K \bigcap\left(\Omega_{d} \backslash \overline{\Omega_{r}}\right), u_{3} \in K \bigcap\left(\Omega_{R} \backslash \overline{\Omega_{d}}\right)$.

According to lemma 3, we have $u_{1} \neq 0$. It means that problem (1) exits at least three positive solutions $u_{1}, u_{2}, u_{3}$, and they satisfy

$$
0<\left\|u_{1}\right\|_{[-\tau, T]}<r<\left\|u_{2}\right\|_{[-\tau, T]}<d<\left\|u_{3}\right\|_{[-\tau, T]}<R
$$

\section{References}

Bainov, D. D., \& Hristova, S. G. (1993). Application of Lakshmikantham's monotone-iterative technique to the solution of the initial value problem for impulse integro-differential equations. J. Appl. Math. Stoch. Anal. 6, 25-34. http://dx.doi.org/10.1155/S1048953393000036

Bainov, D. D., \& Simeonov, P. S. (1993). Impulsive Differential Equations: Periodic Solutions and Applications. Longman Scientific and Technical, Harlow, UK.

Ding, W., Mi, J., \& Han, M. (2005). periodic boundary value problems for first order impulsive functional differential equations. Appl. Math. Comput., 165, 433-446. http://dx.doi.org/10.1016/j.amc.2004.06.022

He, Z., \& He, X. (2004). Periodic boundary value problem for first order impulsive integro-differential equations of mixed type. Journal of Mathematical Analysis and Applications, 296(1), 8-20. http://dx.doi.org/10.1016/j.jmaa.2003.12.047

Lakshmikantham, V., Bainov, D. D., \& Simeonov, P. S. (1989). Theory of Impulsive Differential Equations, Vol. 6 of Series in Modern Applied Mathematics, World Scientific, Teaneck, NJ, USA.

Luo, Z., \& Jing, J. (2008). Periodic boundary value problem for first order impulsive functional differential equations. Computers $\mathcal{E}$ Mathematics with Applications, 55(9), 2090-2107. 
Juan, J. N., \& Rosana, R. L. (2006). Periodic boundary value problem for non-Lipschitzian impulsive functional differential equations. Journal of Mathematical Analysis and Applications, 318(2), 593-610. http://dx.doi.org/10.1016/j.jmaa.2005.06.014

Zhang, K., \& Liu, X. (2010). Nonlinear boundary value problem of first- order impulsive functional differential equations. Journal of Inequalities and Applications, 1-14. http://dx.doi.org/10.1155/2010/490741

Zhang, X., Li, X., Jiang, D., \& Wang, K. (2006). Multiplicity positive solutions to periodic problems for first-order impulsive differential equations. Computers $\mathcal{E}$ Mathematics with Applications, 52(6-7), 953-966.

Zhao, Y. L. (2010). Existence and multiplicity of positive solutions for non-linear differential equations. [Online] Available: http://d.g.wanfangdata.com.cn/Thesis_Y1538875.aspx

Zhimin, Z., \& Weigao G. (2002). Monotone iterative technique and periodic boundary value problem for first order impulsive functional differential equations. Acta Mathematica Sinica, 18, 253-262. http://dx.doi.org/10.1007/s101140200155

Zhimin, Z., \& She, Y. J. (2002). Periodic boundary value problem for first-order impulsive ordinary differential equations. Journal of Mathematical Analysis and Applications, 272, 67-78. http://dx.doi.org/10.1016/S0022-247X(02)00133-6 